\title{
Stability Analysis of Nonlinear Systems with Slope Restricted Nonlinearities
}

\author{
Xian Liu, Jiajia Du, and Qing Gao \\ Key Lab of Industrial Computer Control Engineering of Hebei Province, Institute of Electrical Engineering, Yanshan University, \\ Qinhuangdao 066004, China
}

Correspondence should be addressed to Xian Liu; liuxian@ysu.edu.cn

Received 22 August 2013; Accepted 14 November 2013; Published 28 January 2014

Academic Editors: H. Cakalli and S. Mohiuddine

Copyright (C) 2014 Xian Liu et al. This is an open access article distributed under the Creative Commons Attribution License, which permits unrestricted use, distribution, and reproduction in any medium, provided the original work is properly cited.

The problem of absolute stability of Lur'e systems with sector and slope restricted nonlinearities is revisited. Novel time-domain and frequency-domain criteria are established by using the Lyapunov method and the well-known Kalman-Yakubovich-Popov (KYP) lemma. The criteria strengthen some existing results. Simulations are given to illustrate the efficiency of the results.

\section{Introduction}

Absolute stability of nonlinear systems has been investigated comprehensively for the past several decades [1-12]. It is well known that the Popov criterion and the circle criterion are two classical results with the forms of frequency-domain inequalities (FDIs), which are turned out to be equivalent to some linear matrix inequalities (LMIs). This not only gives the opportunity to use the powerful LMI toolbox [13] to study absolute stability, but also gives the opportunity to consider the controller design problems. In [14], absolute stability of single-input and single-output Lur'e systems with a sector and slope restricted nonlinearity is brought forward. It is pointed out that the slope restriction on the nonlinearity strengthens the Popov criterion by adding an additional term to the original FDI of the criterion. Much work [15-22] on the slope restricted and multivariable problem has been done by using a Lure-Postnikov function or an extended Lur'e-Postnikov function.

In this paper, both time-domain criterion and frequencydomain criterion for absolute stability of Lur'e systems with sector and slope restricted nonlinearities are presented based on the Lyapunov method and the KYP lemma. Some mathematical tools are used through the derivation of the absolute stability criterion. Compared with some existing results, the proposed results are less conservative. This should be owed to the effect of the slope restricted conditions on the nonlinearities. The rest of the paper is organized as follows. In Section 2, the system description and some preliminaries are presented. Time-domain and frequency-domain criteria for absolute stability of the system are given in Section 3. Numerical examples are given in Section 4 and some concluding remarks are given in Section 5.

Throughout this paper, the superscript $*$ means transpose of real matrices and conjugate transpose of complex matrices. For a Hermitian matrix $W, W>0(W \geq 0)$ denotes that $W$ is a positive definite (semidefinite) matrix and $W<0$ denotes that $W$ is a negative definite matrix. $\operatorname{Re}\{Y\}$ means $(1 / 2)(Y+$ $Y^{*}$ ) for any real or complex square matrix $Y$.

\section{Problem Statement}

Consider the following multi-input and multioutput Lur'e system

$$
\begin{gathered}
\dot{x}(t)=A x(t)+B \varphi(\sigma(t)), \\
\sigma(t)=C^{*} x(t),
\end{gathered}
$$

where $A \in \mathbb{R}^{n \times n}, B \in \mathbb{R}^{n \times m}$, and $C \in \mathbb{R}^{n \times m}$ are real matrices, $\varphi(0)=0, \sigma(t)=\left[\begin{array}{c}\sigma_{1}(t) \\ \vdots \\ \sigma_{m}(t)\end{array}\right]$ is the output, $\varphi(\sigma(t))=\left[\begin{array}{c}\varphi_{1}\left(\sigma_{1}(t)\right) \\ \vdots \\ \varphi_{m}\left(\sigma_{m}(t)\right)\end{array}\right]$ 
is piecewise continuously differentiable on $\mathbb{R}^{m}$, and $\varphi_{i}\left(\sigma_{i}(t)\right)(i=1,2, \ldots, m)$ are assumed to satisfy

$$
\begin{gathered}
\gamma_{1 i} \sigma_{i}^{2}(t) \leq \varphi_{i}\left(\sigma_{i}(t)\right) \sigma_{i}(t) \leq \delta_{1 i} \sigma_{i}^{2}(t), \\
\gamma_{2 i} \leq \frac{d \varphi_{i}\left(\sigma_{i}(t)\right)}{d \sigma_{i}(t)} \leq \delta_{2 i},
\end{gathered}
$$

where $\gamma_{2 i} \leq \gamma_{1 i}, \delta_{2 i} \geq \delta_{1 i}, \gamma_{2 i} \leq 0$, and $\delta_{2 i} \geq 0$. The inequalities (2) and (3) denote sector restriction and slope restriction on $\varphi(\sigma(t))$, respectively. Let $\Gamma_{1}=\operatorname{diag}\left(\gamma_{11}, \ldots, \gamma_{1 m}\right)$, $\Delta_{1}=\operatorname{diag}\left(\delta_{11}, \ldots, \delta_{1 m}\right), \Gamma_{2}=\operatorname{diag}\left(\gamma_{21}, \ldots, \gamma_{2 m}\right), \Delta_{2}=$ $\operatorname{diag}\left(\delta_{21}, \ldots, \delta_{2 m}\right)$. Then $\Gamma_{2}-\Gamma_{1} \leq 0, \Delta_{2}-\Delta_{1} \geq 0, \Gamma_{2} \leq 0$, and $\Delta_{2} \geq 0$. Setting $\psi_{i}\left(\sigma_{i}(t)\right)=d \varphi_{i}\left(\sigma_{i}(t)\right) / d t$, (3) is formulated as follows:

$$
\gamma_{2 i} \leq \frac{\psi_{i}\left(\sigma_{i}(t)\right)}{\dot{\sigma}_{i}(t)} \leq \delta_{2 i} .
$$

The transfer function from $\varphi(\sigma(t))$ to $-\sigma(t)$ is denoted as $\chi(s)=C^{*}(A-s I)^{-1} B$.

System (1) is called to be absolutely stable if the equilibrium point $x(t)=0$ is globally asymptotically stable for all nonlinear vector valued functions $\varphi(\sigma(t))$ satisfying (2) and (3). In the following sections, less conservative absolute stability criteria including time-domain criterion and frequency-domain criterion for system (1) are given. Before studying these problems, first we introduce the KYP lemma and Schur complement. These lemmas will be used repeatedly in this paper to get our main results.

Lemma 1 (KYP lemma [23]). Given that $A \in \mathbb{R}^{n \times n}, B \in \mathbb{R}^{n \times m}$, and symmetric matrix $\Sigma \in \mathbb{R}^{(n+m) \times(n+m)}$, with $\operatorname{det}(j \omega I-A) \neq 0$ for $\omega \in \mathbb{R}$, and the pair $(A, B)$ is controllable, the following two statements are equivalent.

(i) $\left[\begin{array}{c}(j \omega I-A)^{-1} B \\ I\end{array}\right]^{*} \Sigma\left[\begin{array}{c}(j \omega I-A)^{-1} B \\ I\end{array}\right] \leq 0$, for all $\omega \in \mathbb{R}$.

(ii) There exists a matrix $P=P^{*}$ such that $\left[\begin{array}{cc}A^{*} P+P A & P B \\ B^{*} P & 0\end{array}\right]+$ $\Sigma \leq 0$. The equivalence for strict inequalities holds even if $(A, B)$ is not controllable.

Lemma 2 (Schur complement [24]). The LMI $\left[\begin{array}{cc}S_{11} & S_{12} \\ S_{12}^{*} & -S_{22}\end{array}\right]<0$ is equivalent to one of the following statements:

(i) $S_{22}>0$ and $S_{11}+S_{12} S_{22}^{-1} S_{12}^{*}<0$;

(ii) $S_{11}<0$ and $S_{22}+S_{12}^{*} S_{11}^{-1} S_{12}>0$.

\section{Main Results}

We choose the following Lur'e-Postnikov function:

$$
V(x(t))=x^{*}(t) P x(t)+\sum_{i=1}^{m} \lambda_{i} \int_{0}^{\sigma_{i}(t)} \varphi_{i}(s) d s
$$

as the Lyapunov function, where $P=P^{*}$ and $\lambda_{i} \in \mathbb{R}(i=$ $1,2, \ldots, m)$ are necessary to be determined. It should be pointed out that $P$ is not necessary to be positive definite and $\lambda_{i}(i=1,2, \ldots, m)$ are not necessary to be nonnegative.
Theorem 3. System (1) is absolutely stable for all $\varphi(\sigma(t))$ satisfying (2) and (3) if $A+B \Gamma_{1} C^{*}$ is Hurwitzian and there exist diagonal matrices $\Lambda=\operatorname{diag}\left(\lambda_{1}, \ldots, \lambda_{m}\right), T_{1} \geq 0, T_{2}>0$, and symmetric matrices $P$ such that the LMI is feasible:

$$
\left[\begin{array}{ccc}
A^{*} P+P A+\Sigma_{11} & P B+\Sigma_{12} & \Sigma_{13} \\
B^{*} P+\Sigma_{12}^{*} & \Sigma_{22} & \Sigma_{23} \\
\Sigma_{13}^{*} & \Sigma_{23}^{*} & -T_{2}
\end{array}\right]<0,
$$

where

$$
\begin{gathered}
\Sigma_{11}=-C \Gamma_{1} T_{1} \Delta_{1} C^{*}-A^{*} C \Gamma_{2} T_{2} \Delta_{2} C^{*} A, \\
\Sigma_{12}=\frac{1}{2} A^{*} C \Lambda+\frac{1}{2} C T_{1}\left(\Gamma_{1}+\Delta_{1}\right)-A^{*} C \Gamma_{2} T_{2} \Delta_{2} C^{*} B \\
\Sigma_{13}=\frac{1}{2} A^{*} C T_{2}\left(\Gamma_{2}+\Delta_{2}\right), \quad \Sigma_{23}=\frac{1}{2} B^{*} C T_{2}\left(\Gamma_{2}+\Delta_{2}\right), \\
\Sigma_{22}=\frac{1}{2} \Lambda C^{*} B+\frac{1}{2} B^{*} C \Lambda-T_{1}-B^{*} C \Gamma_{2} T_{2} \Delta_{2} C^{*} B .
\end{gathered}
$$

Proof. We will demonstrate that the given conditions imply the negative definiteness of $\dot{V}(x(t))$ and the positive definiteness of $V(x(t))$.

Taking the derivative of $V(x(t))$ along the trajectory of (1), we have

$\dot{V}(x(t))=x^{*}(t) P \dot{x}(t)+\dot{x}^{*}(t) P x(t)+\varphi^{*}(\sigma(t)) \Lambda C^{*} \dot{x}(t)$.

Conditions (2) and (4) for $\varphi_{i}\left(\sigma_{i}(t)\right)$ are equivalent to

$$
\begin{aligned}
& u_{1 i}\left(x_{i}\right)=\left(\varphi_{i}\left(\sigma_{i}(t)\right)-\gamma_{1 i} \sigma_{i}(t)\right)\left(\varphi_{i}\left(\sigma_{i}(t)\right)-\delta_{1 i} \sigma_{i}(t)\right) \leq 0, \\
& u_{2 i}\left(x_{i}\right)=\left(\psi_{i}\left(\sigma_{i}(t)\right)-\gamma_{2 i} \dot{\sigma}_{i}(t)\right)\left(\psi_{i}\left(\sigma_{i}(t)\right)-\delta_{2 i} \dot{\sigma}_{i}(t)\right) \leq 0 .
\end{aligned}
$$

For any $t_{1 i} \geq 0$ and $t_{2 i}>0, i=1,2, \ldots, m$, it follows

$$
\begin{aligned}
\sum_{i=1}^{m} t_{1 i} u_{1 i}\left(x_{i}\right)= & \varphi^{*}(\sigma(t)) T_{1} \varphi(\sigma(t)) \\
& -\frac{1}{2} \varphi^{*}(\sigma(t))\left(\Gamma_{1}+\Delta_{1}\right) T_{1} C^{*} x \\
& -\frac{1}{2} x^{*} C T_{1}\left(\Gamma_{1}+\Delta_{1}\right) \varphi(\sigma(t)) \\
& +x^{*} C \Gamma_{1} T_{1} \Delta_{1} C^{*} x \leq 0, \\
\sum_{i=1}^{m} t_{2 i} u_{2 i}\left(x_{i}\right)= & \psi^{*}(\sigma(t)) T_{2} \psi(\sigma(t)) \\
& -\frac{1}{2} \psi^{*}(\sigma(t))\left(\Gamma_{2}+\Delta_{2}\right) T_{2} C^{*} \dot{x} \\
& -\frac{1}{2} \dot{x}^{*} C T_{2}\left(\Gamma_{2}+\Delta_{2}\right) \psi(\sigma(t)) \\
& +\dot{x}^{*} C \Gamma_{2} T_{2} \Delta_{2} C^{*} \dot{x} \leq 0,
\end{aligned}
$$


where $T_{1}=\operatorname{diag}\left(t_{11}, \ldots, t_{1 m}\right) \geq 0$ and $T_{2}=\operatorname{diag}\left(t_{21}, \ldots\right.$, $\left.t_{2 m}\right)>0$. Then

$$
\begin{aligned}
\dot{V}(x(t)) \leq & x^{*}(t) P \dot{x}(t)+\dot{x}^{*}(t) P x(t)+\varphi^{*}(\sigma(t)) \Lambda C^{*} \dot{x}(t) \\
& -\sum_{i=1}^{m} t_{1 i} u_{1 i}\left(x_{i}\right)-\sum_{i=1}^{m} t_{2 i} u_{2 i}\left(x_{i}\right) .
\end{aligned}
$$

The given condition (6) guarantees the negative definiteness of the right hand of (11). Consequently, $\dot{V}(x(t))$ is negative definite.

Now we are only left to demonstrate that $V(x(t))$ is positive definite. In (5), $P$ is only a symmetric matrix but not a positive definite matrix and $\lambda_{i}$ may be a positive or negative number. Therefore, the proof of the positive definiteness of $V(x(t))$ is a little difficult and complex. Without loss of generality, letting $\lambda_{i}<0(i=1,2, \ldots, k)$ and $\lambda_{i} \geq 0(i=$ $k+1, \ldots, m)(0 \leq k \leq m)$, then $V(x(t))$ has the following form:

$$
\begin{aligned}
V(x(t))= & x^{*}(t) P x(t)+\sum_{i=1}^{m} \lambda_{i} \int_{0}^{\sigma_{i}(t)}\left(\gamma_{1 i} s+\varphi_{i}(s)-\gamma_{1 i} s\right) d s \\
\geq & x^{*}(t)\left[P+\frac{1}{2} C \Lambda \Gamma_{1} C^{*}+\frac{1}{2} C \Lambda\left(\Delta_{1 k}-\Gamma_{1 k}\right) C^{*}\right] x(t) \\
& +\sum_{i=k+1}^{m} \lambda_{i} \int_{0}^{\sigma_{i}(t)}\left(\varphi_{i}(s)-\gamma_{1 i} s\right) d s,
\end{aligned}
$$

where $\Delta_{1 k}=\operatorname{diag}\left(\delta_{11}, \ldots, \delta_{1 k}, 0, \ldots, 0\right)$ and $\Gamma_{1 k}=\operatorname{diag}\left(\gamma_{11}\right.$, $\left.\ldots, \gamma_{1 k}, 0, \ldots, 0\right)$. Since (2) implies $\sigma_{i}(t)\left(\varphi_{i}\left(\sigma_{i}(t)\right)-\gamma_{1 i} \sigma_{i}(t)\right) \geq$ $0, \sum_{i=k+1}^{m} \lambda_{i} \int_{0}^{\sigma_{i}(t)}\left(\varphi_{i}(s)-\gamma_{1 i} s\right) d s \geq 0$ is satisfied. Then $V(x(t))$ is positive definite if $P+(1 / 2) C \Lambda \Gamma_{1} C^{*}+(1 / 2) C \Lambda\left(\Delta_{1 k}-\Gamma_{1 k}\right) C^{*}$ is positive definite, which is proved in what follows.

Denote $A_{1}=A+B \Gamma_{1} C^{*}, P_{1}=P+(1 / 2) C \Lambda \Gamma_{1} C^{*}, A_{k}=$ $A_{1}+B\left(\Delta_{1 k}-\Gamma_{1 k}\right) C^{*}$, and $P_{k}=P_{1}+(1 / 2) C \Lambda\left(\Delta_{1 k}-\Gamma_{1 k}\right) C^{*}$. Firstly, the given conditions imply that $A+B \Gamma_{1} C^{*}+B \widetilde{\Delta} C^{*}$ is Hurwitzian for any diagonal matrix $\widetilde{\Delta}$ satisfying $0 \leq \widetilde{\Delta} \leq$ $\Delta_{1}-\Gamma_{1}$. Actually, the matrix $A+B \Gamma_{1} C^{*}+B \widetilde{\Delta} C^{*}$ is Hurwitzian for $\widetilde{\Delta}=0$ in virtue of the given conditions. So we will demonstrate that $A+B \Gamma_{1} C^{*}+B \widetilde{\Delta} C^{*}$ is Hurwitzian for any diagonal matrix $\widetilde{\Delta}$ satisfying $0<\widetilde{\Delta} \leq \Delta_{1}-\Gamma_{1}$. We assume there exists a diagonal matrix $\widetilde{\Delta}$ satisfying $0<\widetilde{\Delta} \leq \Delta_{1}-\Gamma_{1}$ such that the matrix $A+B \Gamma_{1} C^{*}+B \widetilde{\Delta} C^{*}=A_{1}+B \widetilde{\Delta} C^{*}$ is not Hurwitzian. On the one hand, a number $\alpha$ satisfying $0<\alpha \leq 1$ can be found such that

$$
\begin{aligned}
& \operatorname{det}\left(j \omega_{0} I-A_{1}-\alpha B \widetilde{\Delta} C^{*}\right) \\
& \quad=\operatorname{det}\left(j \omega_{0} I-A_{1}\right) \operatorname{det}\left(I-\alpha C^{*}\left(j \omega_{0} I-A_{1}\right)^{-1} B \widetilde{\Delta}\right)=0
\end{aligned}
$$

holds for certain $\omega_{0} \in \mathbb{R}$. Since $A_{1}$ is Hurwitzian, $\operatorname{det}\left(j \omega_{0} I-\right.$ $\left.A_{1}\right) \neq 0$ and $\operatorname{det}\left(I-\alpha C^{*}\left(j \omega_{0} I-A_{1}\right)^{-1} B \widetilde{\Delta}\right)=0$ are followed. The latter formula indicates that there exists a vector $\nu \neq 0$ such that

$$
\nu^{*}\left(I-\alpha \widetilde{\Delta} G^{*}\left(j \omega_{0}\right)\right)=0
$$

where $v^{*} \widetilde{\Delta} \neq 0$ and $G\left(j \omega_{0}\right)=C^{*}\left(j \omega_{0} I-A_{1}\right)^{-1} B$. Then we derive

$$
\begin{aligned}
\nu^{*} \widetilde{\Delta}\{ & T_{1}+\frac{1}{2}\left(\Delta_{1}-\Gamma_{1}\right) T_{1} G\left(j \omega_{0}\right)+\frac{1}{2} G^{*}\left(j \omega_{0}\right) T_{1}\left(\Delta_{1}-\Gamma_{1}\right) \\
& +\frac{1}{2} j \omega_{0} \Lambda G\left(j \omega_{0}\right)+\frac{1}{2}\left[j \omega_{0} \Lambda G\left(j \omega_{0}\right)\right]^{*} \\
& \left.-\omega_{0}^{2} G^{*}\left(j \omega_{0}\right) \Gamma_{2} T_{2} \Delta_{2} G\left(j \omega_{0}\right)\right\} \widetilde{\Delta} \nu \geq 0 .
\end{aligned}
$$

On the another hand, pre- and postmultiplying both sides of (6) by $W_{1}=\left[\begin{array}{ccc}I & C \Gamma_{1} & 0 \\ 0 & I & 0 \\ 0 & 0 & I\end{array}\right]$ and $W_{1}^{*}$, we have

$$
\left[\begin{array}{ccc}
A_{1}^{*} P_{1}+P_{1} A_{1}-A_{*}^{*} C \Gamma_{2} T_{2} \Delta_{2} C^{*} A_{1} & \bar{\Sigma}_{12} & \bar{\Sigma}_{13} \\
\bar{\Sigma}_{12}^{*} & \Sigma_{22} & \Sigma_{23} \\
\bar{\Sigma}_{13}^{*} & \Sigma_{23}^{*} & -T_{2}
\end{array}\right]<0,
$$

where

$$
\begin{gathered}
\bar{\Sigma}_{12}=P_{1} B+\frac{1}{2} A_{1}^{*} C \Lambda+\frac{1}{2} C T_{1}\left(\Delta_{1}-\Gamma_{1}\right)-A_{1}^{*} C \Gamma_{2} T_{2} \Delta_{2} C^{*} B, \\
\bar{\Sigma}_{13}=\frac{1}{2} A_{1}^{*} C T_{2}\left(\Gamma_{2}+\Delta_{2}\right) .
\end{gathered}
$$

By the Schur complement, (16) implies

$$
\left[\begin{array}{cc}
A_{1}^{*} P_{1}+P_{1} A_{1}-A_{1}^{*} C \Gamma_{2} T_{2} \Delta_{2} C^{*} A_{1} & \bar{\Sigma}_{12} \\
\bar{\Sigma}_{12}^{*} & \Sigma_{22}
\end{array}\right]<0 .
$$

From the KYP lemma, we derive that (18) holds if and only if

$$
\left[\begin{array}{c}
\left(j \omega I-A_{1}\right)^{-1} B \\
I
\end{array}\right]^{*}\left[\begin{array}{cc}
\widehat{\Sigma}_{11} & \widehat{\Sigma}_{12} \\
\widehat{\Sigma}_{12}^{*} & \Sigma_{22}
\end{array}\right]\left[\begin{array}{c}
\left(j \omega I-A_{1}\right)^{-1} B \\
I
\end{array}\right]<0,
$$

$\forall \omega \in \mathbb{R}$,

where $\widehat{\Sigma}_{11}=-A_{1}^{*} C \Gamma_{2} T_{2} \Delta_{2} C^{*} A_{1}$ and $\widehat{\Sigma}_{12}=(1 / 2) A_{1}^{*} C \Lambda+$ $(1 / 2) C T_{1}\left(\Delta_{1}-\Gamma_{1}\right)-A_{1}^{*} C \Gamma_{2} T_{2} \Delta_{2} C^{*} B$. Inequality (19) is equivalent to

$$
\begin{aligned}
-T_{1}+ & \frac{1}{2}\left(\Delta_{1}-\Gamma_{1}\right) T_{1} G(j \omega)+\frac{1}{2} G^{*}(j \omega) T_{1}\left(\Delta_{1}-\Gamma_{1}\right) \\
& +\frac{1}{2} j \omega \Lambda G(j \omega)+\frac{1}{2}[j \omega \Lambda G(j \omega)]^{*} \\
& -\omega^{2} G^{*}(j \omega) \Gamma_{2} T_{2} \Delta_{2} G(j \omega)<0, \quad \forall \omega \in \mathbb{R}
\end{aligned}
$$

in terms of the equalities $G(j \omega)=C^{*}\left(j \omega I-A_{1}\right)^{-1} B$ and $j \omega G(j \omega)=C^{*} A_{1}\left(j \omega I-A_{1}\right)^{-1} B+C^{*} B$. Letting $\omega=\omega_{0}$ in 
(20) and pre- and postmultiplying both sides of the resulting inequality by $\nu^{*} \widetilde{\Delta}$ and $\widetilde{\Delta} \nu$, it follows that

$$
\begin{aligned}
v^{*} \widetilde{\Delta} & \left\{-T_{1}+\frac{1}{2}\left(\Delta_{1}-\Gamma_{1}\right) T_{1} G\left(j \omega_{0}\right)\right. \\
& +\frac{1}{2} G^{*}\left(j \omega_{0}\right) T_{1}\left(\Delta_{1}-\Gamma_{1}\right)+\frac{1}{2} j \omega_{0} \Lambda \\
& \times G\left(j \omega_{0}\right)+\frac{1}{2}\left[j \omega_{0} \Lambda G\left(j \omega_{0}\right)\right]^{*} \\
& \left.-\omega_{0}^{2} G^{*}\left(j \omega_{0}\right) \Gamma_{2} T_{2} \Delta_{2} G\left(j \omega_{0}\right)\right\} \widetilde{\Delta} v \\
= & v^{*} \widetilde{\Delta} T_{1}\left[\frac{1}{\alpha}\left(\Delta_{1}-\Gamma_{1}\right)-\widetilde{\Delta}\right] \nu+\frac{\omega_{0}^{2}}{\alpha^{2}} v^{*}\left(-\Gamma_{2} T_{2} \Delta_{2}\right) v<0 .
\end{aligned}
$$

We can observe that (15) and (21) are contradictive, which means that the assumption is not true and $A+B \Gamma_{1} C^{*}+B \widetilde{\Delta} C^{*}$ is Hurwitzian for any diagonal matrix $\widetilde{\Delta}$ satisfying $0 \leq \widetilde{\Delta} \leq$ $\Delta_{1}-\Gamma_{1}$. Therefore, the matrix $A_{k}=A+B \Gamma_{1} C^{*}+B\left(\Delta_{1 k}-\right.$ $\left.\Gamma_{1 k}\right) C^{*}$ is Hurwitzian. Secondly, the given conditions imply that $P+(1 / 2) C \Lambda \Gamma_{1} C^{*}+(1 / 2) C \Lambda\left(\Delta_{1 k}-\Gamma_{1 k}\right) C^{*}$ is positive definite. Actually, pre- and postmultiplying both sides of (16) by $W_{2}=\left[\begin{array}{ccc}I & C\left(\Delta_{1 k}-\Gamma_{1 k}\right) & 0 \\ 0 & I & 0 \\ 0 & 0 & I\end{array}\right]$ and $W_{2}^{*}$ yield

$$
\left[\begin{array}{ccc}
\Xi_{11} & \Xi_{12} & \Xi_{13} \\
\Xi_{12}^{*} & \Sigma_{22} & \Sigma_{23} \\
\Xi_{13}^{*} & \Sigma_{23}^{*} & -T_{2}
\end{array}\right]<0
$$

where

$$
\begin{aligned}
\Xi_{11}= & A_{k}^{*} P_{k}+P_{k} A_{k}+C\left(\Delta_{1 k}-\Gamma_{1 k}\right) \\
& \times T_{1}\left[\left(\Delta_{1}-\Gamma_{1}\right)-\left(\Delta_{1 k}-\Gamma_{1 k}\right)\right] C^{*} \\
& -A_{k}^{*} C \Gamma_{2} T_{2} \Delta_{2} C^{*} A_{k}, \\
\Xi_{12}= & P_{k} B+\frac{1}{2} A_{k}^{*} C \Lambda-A_{k}^{*} C \Gamma_{2} T_{2} \Delta_{2} C^{*} B \\
& +\frac{1}{2} C T_{1}\left(\Delta_{1}-\Gamma_{1}\right)-C T_{1}\left(\Delta_{1 k}-\Gamma_{1 k}\right), \\
\Xi_{13}= & \frac{1}{2} A_{k}^{*} C T_{2}\left(\Gamma_{2}+\Delta_{2}\right) .
\end{aligned}
$$

Inequality (22) implies $\Xi_{11}<0$. According to $0 \leq \Delta_{k}-\Gamma_{k} \leq$ $\Delta_{1}-\Gamma_{1}, T_{2}>0, \Gamma_{2} \leq 0, \Delta_{2} \geq 0, A_{k}^{*} P_{k}+P_{k} A_{k}<0$ is followed. The matrix $A_{k}$ is Hurwitzian, which results in the positive definiteness of $P_{k}$ and $V(x(t))$. This completes the proof.

It is found in the proof of Theorem 3, more exactly in inequality (16), that if (6) holds, then $A+B \Gamma_{1} C^{*}$ is Hurwitzian if and only if $P+(1 / 2) C \Lambda \Gamma_{1} C^{*}>0$.

Theorem 4. System (1) is absolutely stable for all $\varphi(\sigma(t))$ satisfying (2) and (3) if there exist diagonal matrices $\Lambda, T_{1} \geq 0$, $T_{2}>0$, symmetric matrices $P, Q>0$ such that $P+(1 /$ 2) $C \Lambda \Gamma_{1} C^{*}>0$ and the LMI (6) holds.
Remark 5. Theorem 3 is derived directly by using the timedomain method and can be used to study multi-input and multioutput Lur'e systems. Inequality (6) in Theorem 3 is in the form of LMI, which is easier to be solved by means of the LMI toolbox.

The LMI (6) can be transformed into an equivalent FDI. Thus, a frequency-domain criterion for (1) is given as follows.

Theorem 6. System (1) is absolutely stable for all $\varphi(\sigma(t))$ satisfying (2) and (3) if the matrix $A+B \Gamma_{1} C^{*}$ is Hurwitzian and there exist diagonal matrices $\Lambda, T_{1} \geq 0, T_{2}>0$ such that the following frequency-domain inequality holds

$$
\begin{gathered}
\operatorname{Re}\left\{\left[I+\Gamma_{1} \chi(j \omega)\right]^{*} T_{1}\left[I+\Delta_{1} \chi(j \omega)\right]+j \omega \Lambda \chi(j \omega)\right. \\
\left.+\omega^{2}\left[I+\Gamma_{2} \chi(j \omega)\right]^{*} T_{2}\left[I+\Delta_{2} \chi(j \omega)\right]\right\}>0, \\
\omega \in \mathbb{R} .
\end{gathered}
$$

Proof. Let $\bar{P}=\left[\begin{array}{ll}P & 0 \\ 0 & 0\end{array}\right], \bar{A}=\left[\begin{array}{ll}A & B \\ 0 & 0\end{array}\right]$, and $L=\left[\begin{array}{l}0 \\ I\end{array}\right]$. Inequality (6) can be rewritten as

$$
\left[\begin{array}{cc}
\bar{P} \bar{A}+\bar{A}^{*} \bar{P}+\Omega_{11} & \bar{P} L+\Omega_{12} \\
L^{*} \bar{P}+\Omega_{12}^{*} & -T_{2}
\end{array}\right]<0,
$$

where

$$
\Omega_{11}=\left[\begin{array}{cc}
\Sigma_{11} & \Sigma_{12} \\
\Sigma_{12}^{*} & \Sigma_{22}
\end{array}\right], \quad \Omega_{12}=\left[\begin{array}{c}
\Sigma_{13} \\
\Sigma_{23}
\end{array}\right]
$$

According to the KYP lemma, (25) is equivalent to

$$
\left[\begin{array}{c}
(j \omega I-\bar{A})^{-1} L \\
I
\end{array}\right]^{*}\left[\begin{array}{ll}
\Omega_{11} & \Omega_{12} \\
\Omega_{12}^{*} & -T_{2}
\end{array}\right]\left[\begin{array}{c}
(j \omega I-\bar{A})^{-1} L \\
I
\end{array}\right]<0,
$$

$\forall \omega \in \mathbb{R}$.

By simple computations, we have

$$
\begin{aligned}
& (j \omega I-\bar{A})^{-1} L=\frac{1}{j \omega}\left[\begin{array}{c}
(j \omega I-A)^{-1} B \\
I
\end{array}\right], \\
& C^{*} A(A-j \omega I)^{-1} B=C^{*} B+j \omega \chi(j \omega),
\end{aligned}
$$

where $\chi(j \omega)=C^{*}(A-j \omega I)^{-1} B$. Substituting (28) into (27), the equivalence between (6) and (24) is derived.

Remark 7. For the case $\Gamma_{1}=0$, the FDI (24) reduces to

$$
\begin{aligned}
T_{1}+\operatorname{Re}\{ & \left(T_{1} \Delta_{1}+j \omega \Lambda\right) \chi(j \omega)+\omega^{2}\left[I+\Gamma_{2} \chi(j \omega)\right]^{*} \\
& \left.\times T_{2}\left[I+\Delta_{2} \chi(j \omega)\right]\right\}>0, \quad \omega \in \mathbb{R},
\end{aligned}
$$

which corresponds to the FDI as given in Theorem 1.15.1 in [4]. However, the results there only aim at single-input and single-output Lur'e systems.

If the slope restrictions on $\varphi(\sigma(t))$ are removed, another absolute stability criterion is derived by choosing (5) as the Lyapunov function. 
Theorem 8. System (1) is absolutely stable for all $\varphi(\sigma(t))$ satisfying (2) if the matrix $A+B \Gamma_{1} C^{*}$ is Hurwitzian and there exist diagonal matrices $\Lambda, T \geq 0$, symmetric matrices $P, Q>0$, such that the following LMI is feasible:

$$
\left[\begin{array}{cc}
A^{*} P+P A-C \Gamma_{1} T \Delta_{1} C^{*} & P B+\Omega_{12} \\
B^{*} P+\Omega_{12}^{*} & \Omega_{22}
\end{array}\right]<0,
$$

where $\Omega_{12}=(1 / 2) A^{*} C \Lambda+(1 / 2) C T\left(\Gamma_{1}+\Delta_{1}\right), \Omega_{22}=$ $(1 / 2) \Lambda C^{*} B+(1 / 2) B^{*} C \Lambda-T$.

Proof. The proof is similar to that of Theorem 3.

Remark 9. Theorem 8 gives absolute stability conditions for sector restricted Lur'e systems. In fact, the slope restricted condition (3) plays an important role in improving the condition of absolute stability. The forthcoming example shows that Theorem 3 is less conservative than Theorem 8 .

Similar to Theorem 3, an equivalent frequency-domain criterion to Theorem 8 can be given as follows.

Theorem 10. System (1) is absolutely stable for all $\varphi(\sigma(t))$ satisfying (2) if the matrix $A+B \Gamma_{1} C^{*}$ is Hurwitzian and there exist diagonal matrices $\Lambda, T \geq 0$ such that the following FDI holds:

$$
\begin{aligned}
& \operatorname{Re}\left\{\left[I+\Gamma_{1} \chi(j \omega)\right]^{*} T\left[I+\Delta_{1} \chi(j \omega)\right]+j \omega \Lambda \chi(j \omega)\right\}>0, \\
& \omega \in \mathbb{R} .
\end{aligned}
$$

Proof. From the KYP lemma, (30) is equivalent to

$$
\left[\begin{array}{c}
(j \omega I-A)^{-1} B \\
I
\end{array}\right]^{*}\left[\begin{array}{cc}
-C \Gamma_{1} T \Delta_{1} C^{*} & \Omega_{12} \\
\Omega_{12}^{*} & \Omega_{22}
\end{array}\right]\left[\begin{array}{c}
(j \omega I-A)^{-1} B \\
I
\end{array}\right]<0,
$$

$\forall \omega \in \mathbb{R}$.

The equivalence between (30) and (31) is derived from $\chi(j \omega)=C^{*}(A-j \omega I)^{-1} B$ and $C^{*} A(A-j \omega I)^{-1} B=C^{*} B+$ $j \omega \chi(j \omega)$.

Remark 11. Theorem 10 includes two particular cases. For the case $\Lambda=0,(31)$ is reduced to

$$
\operatorname{Re}\left\{\left(I+\Gamma_{1} \chi(j \omega)\right)^{*} T\left(I+\Delta_{1} \chi(j \omega)\right)\right\}>0, \quad \omega \in \mathbb{R}
$$

Correspondingly, Theorem 10 is in the form of the circle criterion. For the case $\Gamma_{1}=0,(31)$ reduces to

$$
T+\operatorname{Re}\left\{\left(j \omega \Lambda+T \Delta_{1}\right) \chi(j \omega)\right\}>0, \quad \omega \in \mathbb{R} .
$$

Theorem 10 has the same form as the Popov criterion.

\section{Numerical Example}

In this section, a numerical example is presented to illustrate the effectiveness of the proposed results.

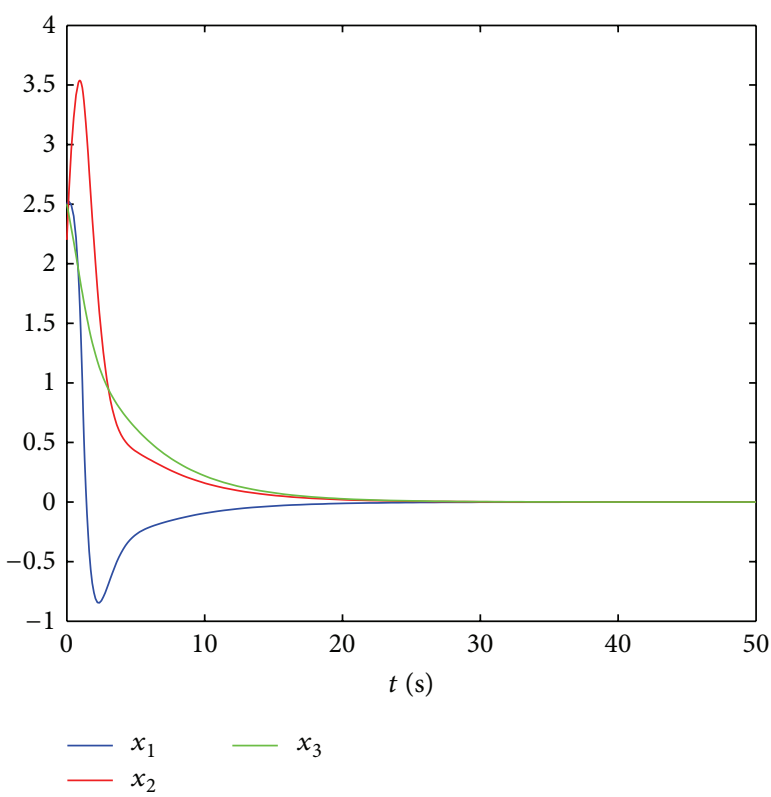

Figure 1: The states of system (35).

Consider Chua's oscillator [25] with the following dimensionless equations

$$
\begin{aligned}
& \dot{x}_{1}(t)=\alpha\left[x_{2}(t)-x_{1}(t)-f\left(x_{1}(t)\right)\right], \\
& \dot{x}_{2}(t)=x_{1}(t)-x_{2}(t)+x_{3}(t), \\
& \dot{x}_{3}(t)=-\beta x_{2}(t)-\gamma x_{3}(t),
\end{aligned}
$$

where $f\left(x_{1}(t)\right)=m_{1} x_{1}(t)+(1 / 2)\left(m_{0}-m_{1}\right)\left(\left|x_{1}(t)+1\right|-\right.$ $\left.\left|x_{1}(t)-1\right|\right), \alpha, \beta, \gamma, m_{0}$, and $m_{1}$ are numbers. System (35) can be reformulated in the form of (1) with $x(t)=\left[\begin{array}{l}x_{1}(t) \\ x_{2}(t) \\ x_{3}(t)\end{array}\right]$, $A=\left[\begin{array}{ccc}-\alpha & \alpha & 0 \\ 1 & -1 & 1 \\ 0 & -\beta & -\gamma\end{array}\right], B=\left[\begin{array}{c}-\alpha \\ 0 \\ 0\end{array}\right], C=\left[\begin{array}{lll}1 & 0 & 0\end{array}\right]^{*}, \sigma(t)=x_{1}(t)$, and $\varphi(\sigma(t))=m_{1} \sigma(t)+(1 / 2)\left(m_{0}-m_{1}\right)(|\sigma(t)+1|-|\sigma(t)-1|)$. The nonlinearity $\varphi(\sigma(t))$ satisfies

$$
\begin{gathered}
\min \left\{m_{0}, m_{1}\right\} \sigma(t)^{2} \leq \varphi(\sigma(t)) \sigma(t) \leq \max \left\{m_{0}, m_{1}\right\} \sigma(t)^{2}, \\
\min \left\{m_{0}, m_{1}\right\} \leq \frac{d \varphi(\sigma(t))}{d \sigma(t)} \leq \max \left\{m_{0}, m_{1}\right\}
\end{gathered}
$$

Thus, $\Gamma_{1}=\Gamma_{2}=\min \left\{m_{0}, m_{1}\right\}$ and $\Delta_{1}=\Delta_{2}=\max \left\{m_{0}, m_{1}\right\}$.

When $\alpha=-0.8018, \beta=0.136, \gamma=0.1097$, and $m_{0}=-2.96$ are taken, system (35) is absolutely stable for $m_{1} \leq 2.009$ by applying Theorem 3 . However, we derive that system (35) is absolutely stable for $m_{1} \leq 1.81$ and $m_{1} \leq$ 1.51, respectively, by Theorem 8 and the Popov criterion. This shows that Theorem 3 is an improvement with respect to Theorem 8 and the Popov criterion, and the slope restrictions could improve the absolute stability condition. The states of system (35) with $m_{1}=2$ at the initial value $\left[\begin{array}{lll}2.5 & 2.2 & 2.5\end{array}\right]^{*}$ are given in Figure 1, from which it is illustrated that system (35) is absolutely stable. 


\section{Conclusion}

We have proposed new absolute stability criteria for Lur'e systems with sector and slope restricted nonlinearities from time-domain and frequency-domain points of view. The slope restrictions on nonlinearities improve the absolute stability conditions. We have shown that the criteria are less conservative than some existing results.

\section{Conflict of Interests}

The authors declare that there is no conflict of interests regarding the publication of this paper.

\section{Acknowledgments}

This research is supported by the National Natural Science Foundation of China $(61004050,61172095)$ and the Natural Science Foundation of Scientific Research of Hebei Education Department (2009482).

\section{References}

[1] V. M. Popov, "Absolute stability of nonlinear systems of automatic control," Automation and Remote Control, vol. 22, no. 8, pp. 857-875, 1961.

[2] L. T. Grujić, "On absolute stability and the aizerman conjecture," Automatica, vol. 17, no. 2, pp. 335-349, 1981.

[3] W. M. Haddad and D. S. Bernstein, "Parameter-dependent Lyapunov functions and the discrete-time Popov criterion for robust analysis," Automatica, vol. 30, no. 6, pp. 1015-1021, 1994.

[4] J. Cao and S. Zhong, "New delay-dependent condition for absolute stability of Lurie control systems with multiple time-delays and nonlinearities," Applied Mathematics and Computation, vol. 194, no. 1, pp. 250-258, 2007.

[5] R. Medina, "Absolute stability of discrete-time systems with delay," Advances in Difference Equations, vol. 2008, Article ID 396504, 10 pages, 2008.

[6] Q. L. Han, "A new delay-dependent absolute stability criterion for a class of nonlinear neutral systems," Automatica, vol. 44, no. 1, pp. 272-277, 2008.

[7] J. Cao, S. Zhong, and Y. Hu, "Delay-dependent condition for absolute stability of Lurie control systems with multiple time delays and nonlinearities," Journal of Mathematical Analysis and Applications, vol. 338, no. 1, pp. 497-504, 2008.

[8] H. Wang, A. Xue, and R. Lu, "Absolute stability criteria for a class of nonlinear singular systems with time delay," Nonlinear Analysis: Theory, Methods and Applications, vol. 70, no. 2, pp. 621-630, 2009.

[9] B. Zhang, J. Lam, S. Xu, and Z. Shu, "Absolute exponential stability criteria for a class of nonlinear time-delay systems," Nonlinear Analysis: Real World Applications, vol. 11, no. 3, pp. 19631976, 2010.

[10] S. M. Lee and J. H. Park, "Delay-dependent criteria for absolute stability of uncertain time-delayed Lur'e dynamical systems," Journal of the Franklin Institute, vol. 347, no. 1, pp. 146-153, 2010.

[11] C. A. C. Gonzaga, M. Jungers, and J. Daafouz, "Stability analysis of discrete-time Lure systems," Automatica, vol. 48, no. 9, pp. 2277-2283, 2012.
[12] D. Wang and F. Liao, "Absolute stability of Lurie direct control systems with time-varying coefficients andmultiple nonlinearities," Applied Mathematics and Computation, vol. 219, no. 9, pp. 4465-4473, 2013.

[13] P. Gahinet, A. Nemirovski, A. J. Laub, and M. Chilali, LMI Control Toolbox Users Guide, The Math Works, Natick, Mass, USA, 1995.

[14] V. A. Yakubocivh, "The method of matrix inequalities in the stability theory of nonlinear control systems: II," Automatica and Telemechanic, vol. 26, pp. 577-592, 1965.

[15] J. A. K. Suykens, J. Vandewalle, and B. de Moor, "An absolute stability criterion for the Lur'e problem with sector and slope restricted nonlinearities," IEEE Transactions on Circuits and Systems I, vol. 45, no. 9, pp. 1007-1009, 1998.

[16] S. M. Lee, J. H. Park, and O. M. Kwon, "Improved asymptotic stability analysis for Lur'e systems with sector and slope restricted nonlinearities," Physics Letters A, vol. 362, no. 5-6, pp. 348-351, 2007.

[17] S. M. Lee, O. M. Kwon, and J. H. Park, "Delay-independent absolute stability for time-delay Lur'e systems with sector and slope restricted nonlinearities," Physics Letters A, vol. 372, no. 22, pp. 4010-4015, 2008.

[18] S. J. Choi, S. M. Lee, S. C. Won, and J. H. Park, "Improved delay-dependent stability criteria for uncertain Lur'e systems with sector and slope restricted nonlinearities and time-varying delays," Applied Mathematics and Computation, vol. 208, no. 2, pp. 520-530, 2009.

[19] S. M. Lee and J. H. Park, "Robust stabilization of discretetime nonlinear Lur'e systems with sector and slope restricted nonlinearities," Applied Mathematics and Computation, vol. 200, no. 1, pp. 429-436, 2008.

[20] D. H. Ji, J. H. Park, and S. C. Won, "Master-slave synchronization of Lur'e systems with sector and slope restricted nonlinearities," Physics Letters A, vol. 373, no. 11, pp. 1044-1050, 2009.

[21] J. Carrasco, W. P. Heath, and A. Lanzon, "Equivalence between classes of multipliers for slope-restrited nonlinearities," Automatica, vol. 49, no. 6, pp. 1732-1740, 2013.

[22] G. A. Leonov, D. V. Ponomarenko, and V. B. Smirnova, Frequency-Domain Methods for Nonlinear Analysis: Theory and Applications, World Scientific, Singapore, 1996.

[23] A. Rantzer, "On the Kalman-Yakubovich-Popov lemma," Systems and Control Letters, vol. 28, no. 1, pp. 7-10, 1996.

[24] S. Boyd, L. E. Ghaoui, E. Feron, and V. Balakrishnan, Linear Matrix Inequality in System and Control Theory, Society for Industrial and Applied Mathematics, Philadelphia, 1994.

[25] R. Martinez-Guerra, D. M. Corona-Fortunio, and J. L. MataMachuca, "Synchronization of chaotic Liouvillian systems: an application to Chua's oscillator," Applied Mathematics and Computation, vol. 219, no. 23, pp. 10934-10944, 2013. 


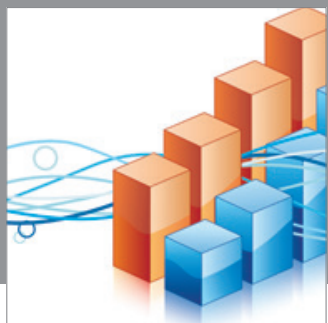

Advances in

Operations Research

mansans

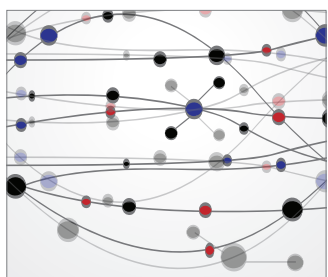

The Scientific World Journal
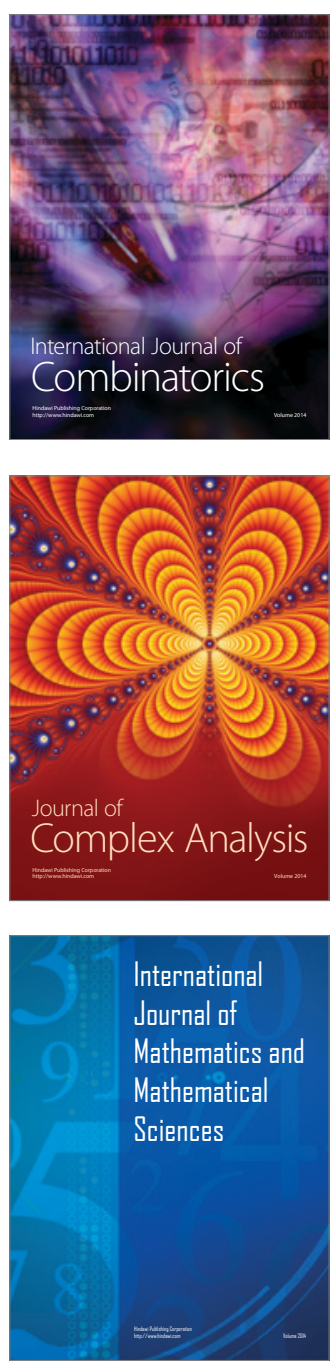
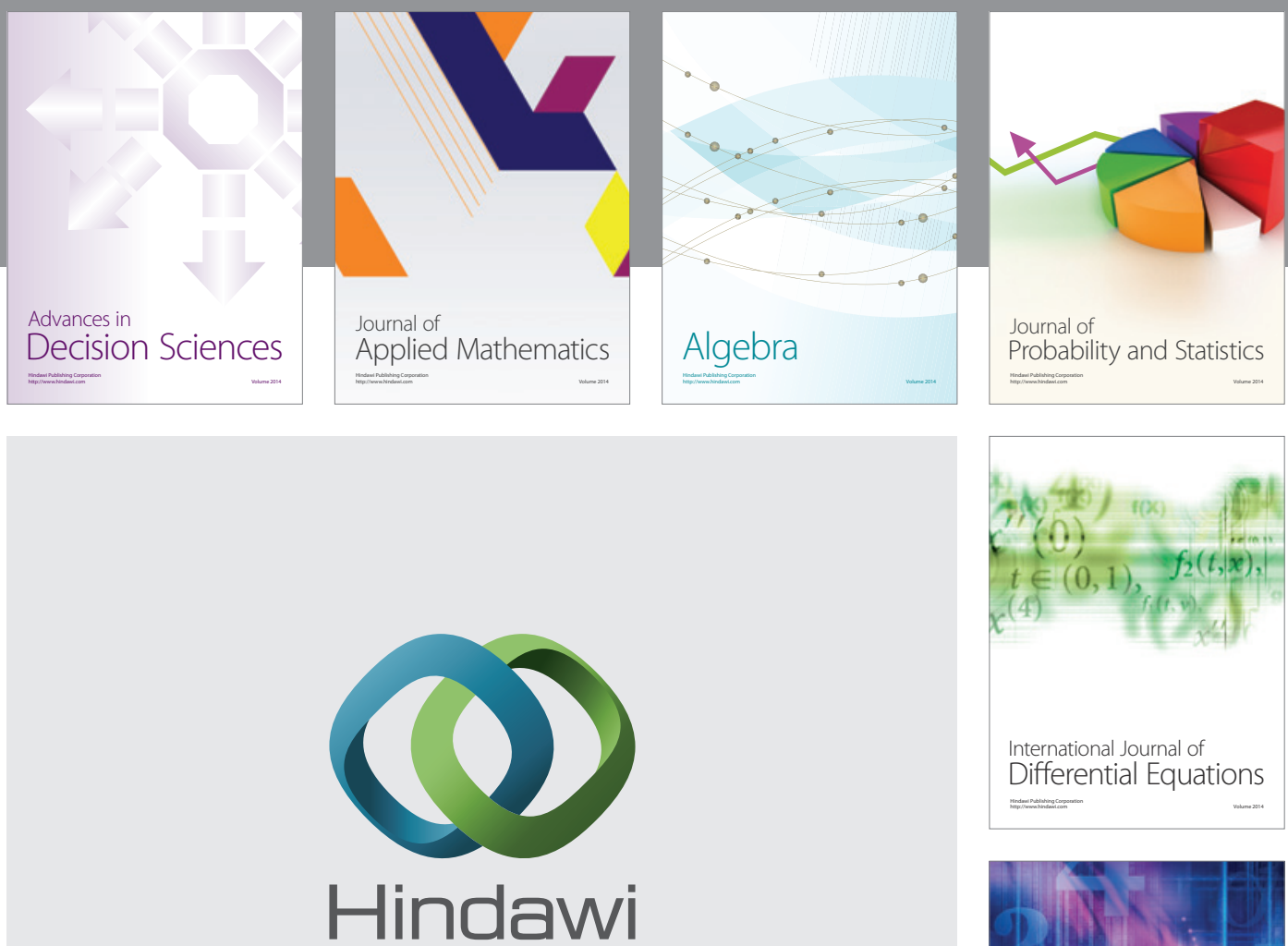

Submit your manuscripts at http://www.hindawi.com
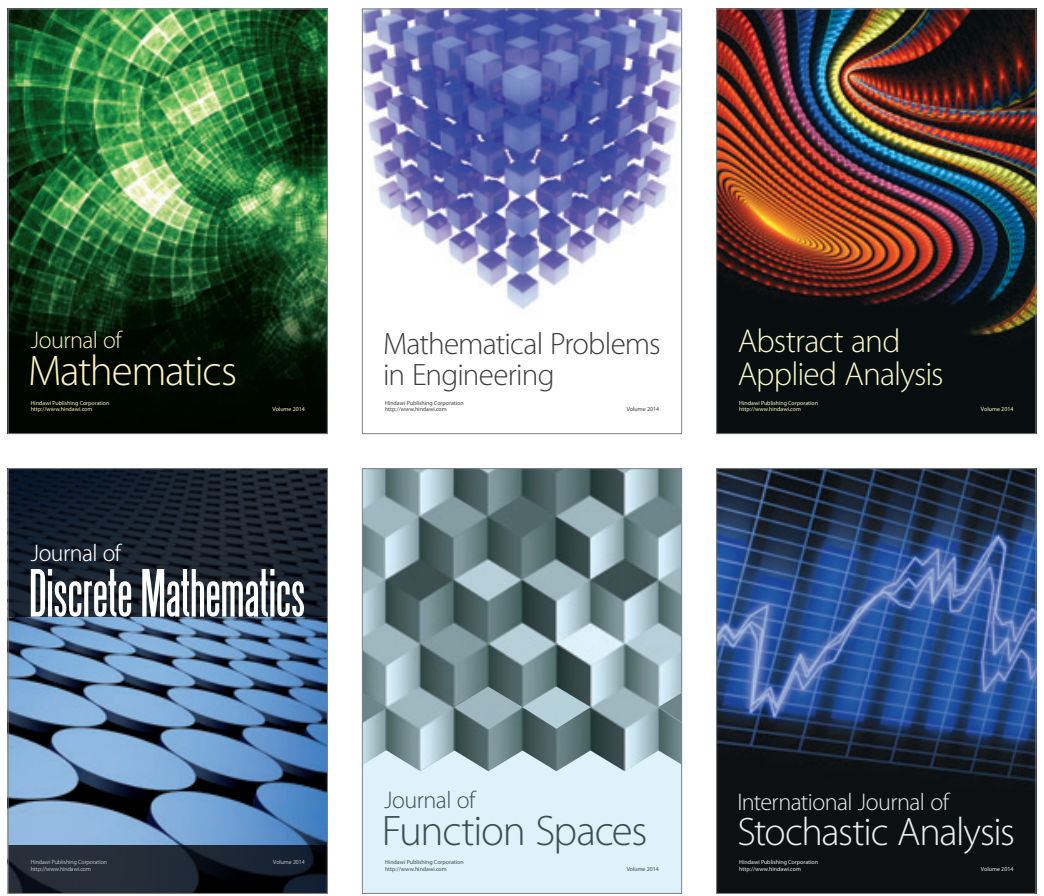

Journal of

Function Spaces

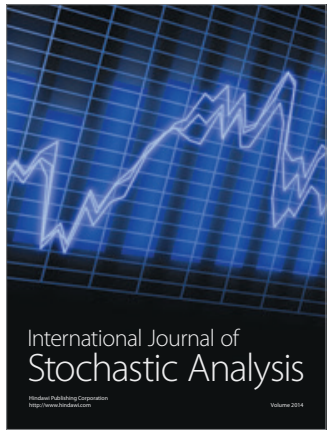

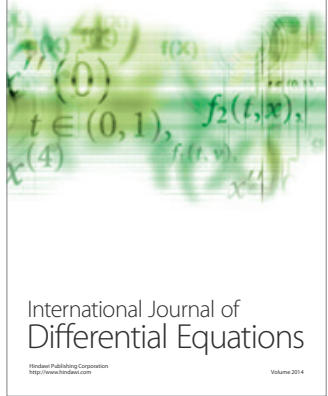
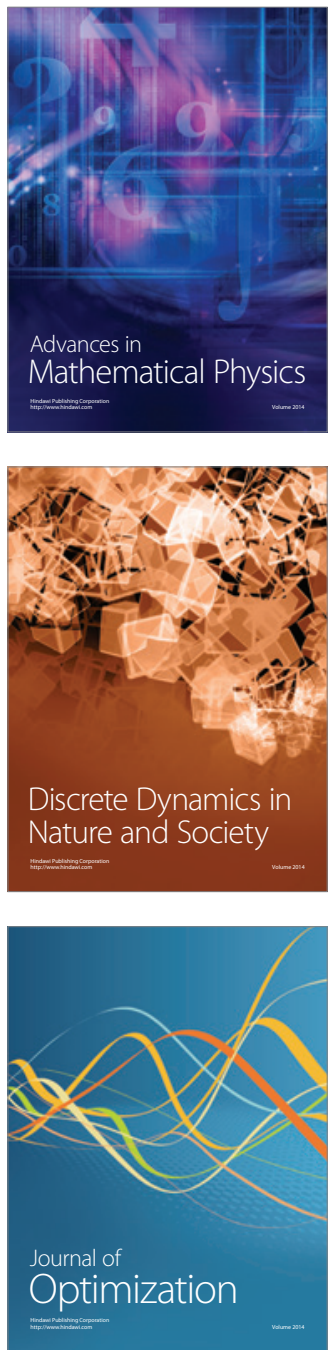